\title{
Multilevel Turbulence Simulations
}

\section{Citation}

Tziperman, Eli, I. Yavneh, and S. Ta'asan. 1993. Multilevel turbulence simulations. Europhysics Letters 24(4): 239-244.

\section{Published Version}

http://dx.doi.org/10.1209/0295-5075/24/4/001

\section{Permanent link}

http://nrs.harvard.edu/urn-3:HUL.InstRepos:3415484

\section{Terms of Use}

This article was downloaded from Harvard University's DASH repository, and is made available under the terms and conditions applicable to Other Posted Material, as set forth at http:// nrs.harvard.edu/urn-3:HUL.InstRepos:dash.current.terms-of-use\#LAA

\section{Share Your Story}

The Harvard community has made this article openly available.

Please share how this access benefits you. Submit a story.

Accessibility 


\title{
Multilevel Turbulence Simulations.
}

\author{
E. Tziperman $(*)$, I. Yavneh $\left({ }^{* *}\right)$ and S. Ta'ASan $(* *)$ \\ (*) Environmental Sciences and Energy Research
}

The Weizmann Institute of Science - Rehovot 76100, Israel

(**) Department of Applied Mathematics, The Weizmann Institute of Science Rehovot 76100, Israel

(received 7 June 1993; accepted in final form 21 September 1993)

PACS. 02.70 - Computational techniques.

PACS. 47.25C - Isotropic turbulence.

PACS. 92.10L - Turbulence, diffusion, and mixing.

\begin{abstract}
A novel multigrid method for the accurate and efficient simulation of turbulent flows is described and demonstrated. The method's efficiency relative to direct simulations is of the order of the ratio of required integration time to the smallest-eddy turnover time, potentially resulting in orders-of-magnitude improvement for a large class of turbulence problems.
\end{abstract}

The numerical simulation of high-Reynolds-number (Re) turbulent flows poses a major challenge because of the large number of degrees of freedom characterizing such flows. Numerical simulations of such flows are divided into direct simulations (DS), in which all scales are resolved, and large eddy simulations (LES), in which only the largest turbulent scales are explicitly simulated, with smaller scales modelled by some closure scheme. While DS are more accurate, they are still limited at present to $R e$ that are significantly lower than what occurs in natural flows for both 2D and 3D turbulence studies [1]. LES, on the other hand, can be used at much higher Re, but their accuracy, of course, depends on the quality of the closure scheme used. Yet, even LES still require prohibitively large computational resources when applied to natural flows, while their accuracy is often not satisfactory [2]. Clearly a novel algorithm for the simulation of turbulent flows is badly needed.

We propose here a novel method for the simulation of turbulent flows, that is motivated by and based on the powerful multigrid (MG) formalism [3]. The method, called «Multilevel Turbulence Simulations» (MTS), is potentially more efficient and more accurate than LES.

Physical basis of the mathematical algorithm. - In many physical problems one is interested in the effects of the small scales on the larger ones, or in a typical realization of the flow, and not in the detailed time history of each small-scale feature. MTS take advantage of the fact that the detailed simulation of small scales is not needed at all times, in order to make the calculation significantly more efficient, while accurately accounting for the effects of the small scales on the larger scale of interest. 
In MTS, models of several resolutions (i.e. number of grid points or Fourier components) are used to represent the turbulent flow. The coarsest grid is referred to as the lowest MG level, and the finest grid is the highest MG level. The model equations in each coarse level incorporate a closure term (roughly corresponding to the " $\tau$ correction" in the MG formalism [3]) that accounts for the effects of the unresolvable scales on that grid. The finerresolution grids are used only for a small portion of the simulation time in order to evaluate the closure terms for the coarser grids, while the coarse-resolution grids are then used to accurately and efficiently calculate the evolution of the larger scales. This clearly implies that MTS should not be applied to problems where the precise location and time history of specific small eddies are needed. Assuming that the resolution (per spatial dimension) of each MTS level is half of that of the next finer level, a coarse grid is 16 times more efficient (for a 3D model) in integrating the model for a given time than the next finer grid, so that the potential saving achieved with several grids is significant, to say the least.

Expected saving. - Consider first a simple turbulence problem where there are two main scales: small energy-containing eddies, and larger slowly evolving mean flows. In this case, two grids will be used and we may regard a «work unit» in MTS as the work needed to integrate the model on the finer level for a time of the order of the eddy turnover time. Now, the integration time and, therefore, the work required in DS, are proportional to the time scale of the slowly evolving mean flows, while MTS requires «a few work units» [3], i.e. work of the order of the eddy turnover time. The saving is therefore of the order of the ratio of the two times, which could be very significant for many turbulence problems. This restricts the application of MTS to applications that require integrating the model to longer times than the eddy turnover time, such as nearly all geophysical applications and most engineering applications. In most turbulence problems there is a continuum of time and space scales, and accordingly many grids will be used in MTS, with efficiency rapidly increasing with the number of grids used.

An interesting related previous effort was carried out in [4], yet not actually following the MG formalism, and concluding that no more than two grids can be used for this purpose. The above discussion implies that, when applied to the right problem, MTS can be used with as many grids as needed, with a significant saving resulting.

Detailed algorithm. - The MTS cycle, denoted MTS $\left(k, \gamma, \eta_{1}^{k}, \eta_{2}^{k}\right)$, is schematically shown in fig. 1 and is defined recursively following the FAS (Full Approximation Scheme) MG algorithm [3]: $a$ ) Once at level $k$, integrate the turbulence model for a time $\eta_{1}^{k}$, and compute a closure scheme for the coarser level $k-1$ (the $\tau$ correction); $b$ ) transfer the model solution (e.g., the velocity $u^{k}$ ) at the end of the integration at level $k$ to the coarser grid at level $k-1$; c) make $\gamma$ MTS cycles at level $k-1$ to update the solution at that level; $d$ ) return to the finer level $k$. Correct the coarse part of the solution on level $k$ with the updated coarse solution obtained by integrating the model on the coarser level $k-1$. The fine portion of the solution on level $k$ remains frozen there during the integration on coarser levels; finally $e$ ), integrate the model on level $k$ for a time $\eta_{2}^{k}$ to obtain the updated solution at level $k$.

The fine-to-coarse transfer from level $k$ to level $k-1$ can be done by simply setting the wave number amplitudes on the coarse model to those of the corresponding wave numbers in the fine-resolution model (using fast Fourier transform (FFT) in a finite-difference model). The coarse-to-fine transfer of the coarse solution $u^{k-1}$ to level $k$ can be similarly defined as transferring the wave numbers represented on level $k-1$ to level $k$, while not changing the amplitudes of the high wave numbers represented on the finer level alone.

During the fine-to-coarse part of the cycle at MTS level $k$ (steps $a$ ) and $b$ ) above), the closure model for level $k-1$ is calculated in the manner described below. In addition, the 


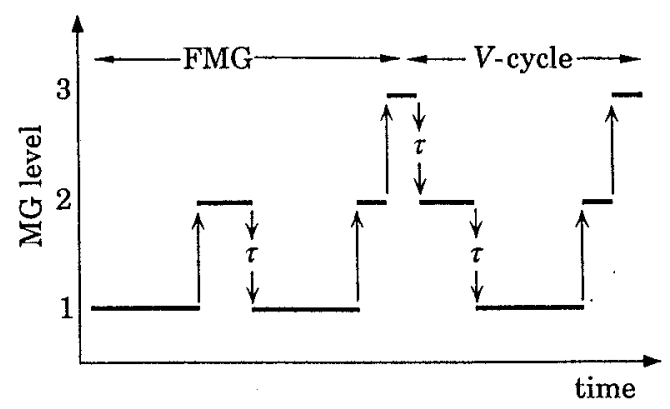

Fig. 1. - Schematic plot of a multigrid cycle in MTS for $\gamma=1$ ( $V$-cycle), including the FMG initialization.

model is stepped in time to efficiently and accurately calculate the evolution of the scales resolved at level $k$.

During the coarse-to-fine part of the cycle (steps $d$ ) and $e$ ) above), the model is run on level $k$ in order to allow the frozen smallest scales resolved on this grid to readjust to the updated larger scales calculated on the previously visited coarser grid $k-1$. This readjustment requires an integration time roughly equal to the eddy turnover time for the smallest eddies represented on level $k$. This minimum integration time on each grid had led [4] to state that only two-level calculations of turbulent flows are feasible, although it is simply a reassessment of the restriction of MTS to integrations that are significantly longer than the eddy turnover time. The freezing of the fine scales between visits to the fine grids is justified by the assumption that the averaged effect of these scales on the larger ones changes over a slow time scale, typical of the large scales. It is sufficient, therefore, to update the small scales only occasionally, once the larger scales have changed to a significant degree. The updating interval depends, of course, on the time scale of the large-scale physics and of the forcing and needs to be chosen very carefully for the results to be accurate. The frozen small scales simply provide reasonable initial conditions that make it easy for these small scales to reach statistical equilibrium with the larger scales during the fine-grid integration.

The $\tau$ correction. - The correct representation of the unresolved subgrid scales at each level by the $\tau$ correction term is perhaps the most crucial part of the MTS algorithm. Trying to simply integrate level $k$ with fixed Reynolds stresses evaluated at level $k+1$ led to numerical instabilities in our numerical experiments. We therefore chose to tune the closure scheme on a given coarse grid using the results of the next finer grid by requiring the simulation on the coarse grid to be accurate. The meaning of «accuracy» here depends on the physical problem and needs to be carefully defined and understood. Due to the inherent unpredictability of turbulent flows, it is only possible to require the accuracy of statistical quantities such as the total energy or enstrophy, as well as the energy or enstrophy spectrum of the flow. When an averaged flow is present, it may be required to be the same on all grids.

For the case of isotropic and homogeneous turbulence considered below as an example, we can calculate the optimal parameter(s) of a closure scheme for level $k$ (e.g., an eddy viscosity, $\left.v^{k}\right)$, that best represent the subgrid scales, by minimizing a cost function which is a measure of the area between the time-averaged spectra of levels $k$ and $k+1$ on a log-log plot of energy 
spectrum vs. wave number

$$
J\left(\nu^{k}\right)=\sum_{q}\left[\log \left\langle E^{k}(q)\right\rangle-\log \left\langle E^{k+1}(q)\right\rangle\right]^{2} \Delta \log q .
$$

The angled brackets here denote a time averaging over a period $T_{\text {avg }}^{k}$, of the order of eddy turnover time for the smallest eddies resolved on level $k ; \Delta \log q=\log (q+1)-\log (q)$; and the sum is over all wave numbers represented on both the $k$ and $k+1$ levels.

The averaged fine-grid spectrum, $\left\langle E^{k+1}(q)\right\rangle$, is calculated during the final stage of the integration on level $k+1$ during the fine-to-coarse part of the MTS cycle. $\left\langle E^{k}(q)\right\rangle$ is calculated by averaging over the same time as $\left\langle E^{k+1}(q)\right\rangle$ and starting from the same initial conditions (as transferred to the coarse grid). Thus, there is a short overlap in the integrations on the fine and coarse grids, used to calculate the $\tau$ correction term. The closure parameters are calculated by repeatedly running the coarse grid $(k)$ with different values of $v^{k}$, until an approximate minimum is reached. These iterations are quite inexpensive, as they involve integrating only the coarse model for a relatively short time. In applying MTS to a given problem, we can use the closure schemes judged best in relevant past LES studies, with the additional advantage that the closure parameters are directly and continuously calculated during the simulation using the results of the fine grids.

Spin-up using FMG initialization. - The initialization of a turbulence simulation to statistical steady state (spin-up) can be rather expensive [2]. In MTS, the spin-up stage is efficiently done using a Full Multigrid cycle (FMG) [3], as seen in fig. 1, by starting the model integration at the coarsest level, and gradually refining the resolution following the usual MG cycling rules.

Computational example. - As a simple, yet far from trivial, example that is intended as a "proof of concept», rather than an application of MTS to a fully complex turbulence problem, consider a model of two-dimensional homogeneous and isotropic turbulence, governed by the $2 \mathrm{D}$ vorticity equation that for level $k$ takes the form

$$
\zeta_{t}^{k}+J\left(\psi^{k}, \zeta^{k}\right)=\mathscr{D}+\mathscr{T}+G^{k}\left(\nu^{k}\right) ; \quad \zeta^{k}=\nabla^{2} \psi^{k},
$$

where $\zeta^{k}$ is the vorticity at level $k, \psi^{k}$ the stream function, $\mathscr{D}$ an energy dissipation term acting as an energy sink at low wave numbers, $\mathscr{T}$ a random steering force at a limited low-wave-number range, and $\sigma^{k}\left(\nu^{k}\right)$ the closure term representing the subgrid scales. Both $\mathscr{T}$ and $\mathscr{D}$ are well represented on the coarsest grid, with their wave number ranges and amplitudes chosen such that no coherent structures develop (Kurtosis values are about 3 for both $\zeta$ and $\psi$ ), and the small scales may therefore be expected to be reasonably well represented in terms of a simple closure scheme. For this $2 \mathrm{D}$ turbulence problem the closure scheme is the anticipated-potential-vorticity method [5]; the cost function measures the difference between enstrophy spectra on the fine and coarse grids; and vorticity is the quantity transferred between the grids (using FFT as explained above). Three MG levels are used: a $64 \times 64$ grid is level 1 , a $128 \times 128$ grid is level 2 , and a $256 \times 256$ grid is level 3 . The minimization used to calculate the closure parameters was stopped within $10 \%$ of the absolute minimum, which typically required five iterations.

The usefulness of the MTS approach will now be demonstrated by showing that it is more efficient than DS, and also accurate in the statistical sense defined above.

Consider two simulations beginning from zero initial conditions, one using a direct simulation with a $256 \times 256$ resolution, and the other following the MTS approach. Figure 2 shows the MG level during the calculation (upper curve). The total enstrophy (middle two 
curves) and total kinetic energy (KE, lower two curves) are also shown for both the MTS calculation (full curves) and the DS (dashed curves). The slow time scale in this problem is of the forcing $\mathscr{T}$, whose amplitude was proportional to $(1-\exp [-t / 15]) \exp [-t / 10]$, while the eddy turnover time here is of the order of about $1 / 3$. The MTS curve is thicker when integration is on the finest level (level 3), where it should be compared to the DS using the same resolution. Clearly, the MTS approach has done quite well in reproducing the DS results. Note that the total enstrophy changes more than the total kinetic energy upon transition between MG levels. That is simply because the enstrophy tends to be concentrated in the small scales, and a smaller portion of it is therefore represented on the coarse grids than for the energy which tends to be concentrated in the larger scales. Measuring the work done in the numerical simulation as the number of time steps times the number of grid points, MTS (including the calculation of the $\tau$ correction) required less than $1 / 6$ of the work required by the DS in this case. This saving could be significantly larger for integrations in which the ratio between the slow time scale and the eddy turnover time is larger.

The importance of the $\tau$ correction is demonstrated in fig. 3, which shows the enstrophy spectrum at time $t=5$ for the DS (solid curve), for MTS with $\tau$ correction (long-dashed curve) and for MTS without the $\tau$ correction (short-dashed curve). Clearly, the MTS calculation with the $\tau$ correction is a better approximation to the DS solution. The case shown without the $\tau$ correction is one with a seemingly reasonable, yet intentionally low, specified value of the eddy viscosity. The fit of the MTS and DS spectra at the lowest wave numbers is not as good as that at higher wave numbers for the following reason. The model is forced at the low wave numbers by random forcing. The flow of KE to low wave numbers in $2 \mathrm{D}$ turbulence results therefore in a strong dependence of the instantaneous lowestwave-number amplitudes on the detailed history of the random force. This history is different, of course, for the DS and MTS simulations, hence the difference in fig. 3 . This
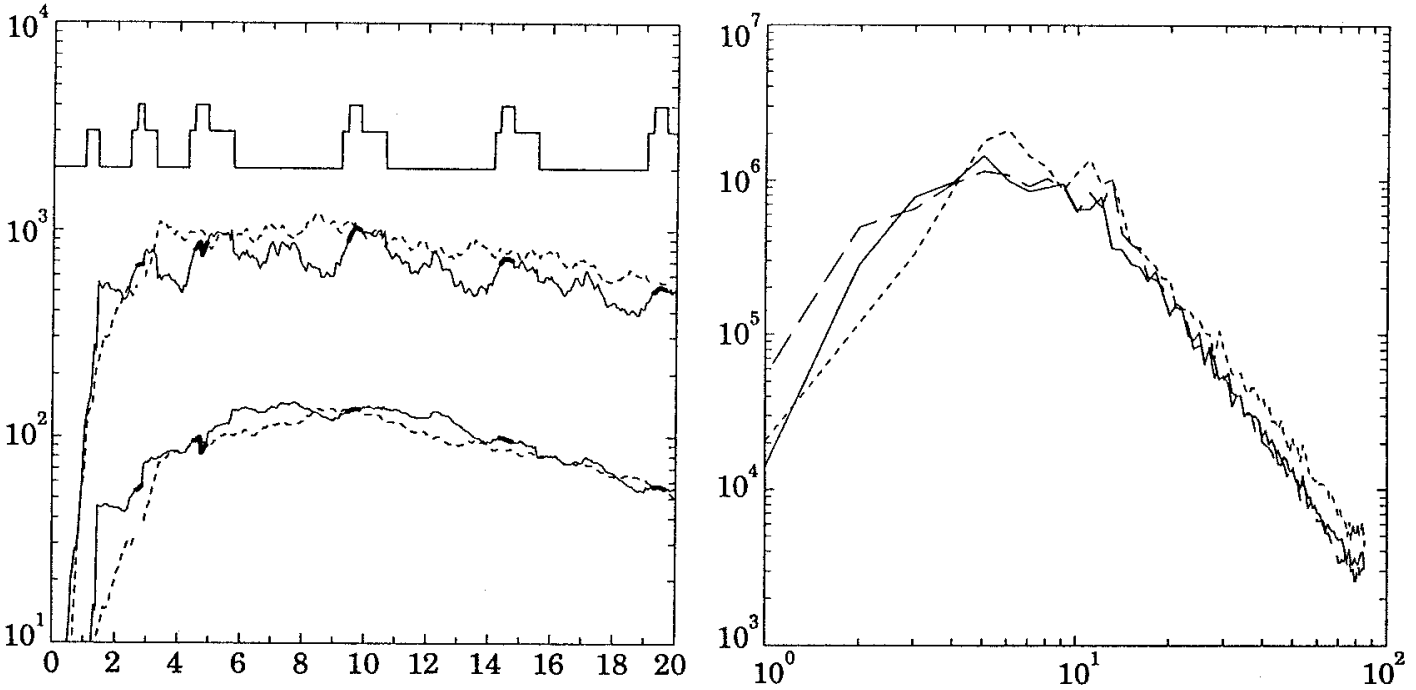

Fig. 2.

Fig. 3

Fig. 2. - Comparing total enstrophy and kinetic energy of a two-dimensional turbulence model using MTS and DS. See text for details.

Fig. 3. - Enstrophy spectra using DS, and MTS with and without $\tau$ correction. See text for details. 
should not be a problem in physical problems with non-random large scale or when comparing; long-term-averaged spectra from DS and MTS.

Conclusions. - We have demonstrated that the Multilevel Turbulence Simulations approach results in an efficient and accurate simulation in the specific problem examined. While the success of this test problem cannot guarantee the success of the MTS approach under more general circumstances, it does indicate very clearly that the method is at least potentially a most useful tool for turbulence studies.

We can see three different situations in which MTS can be useful in turbulence simulations. First, in accelerating the convergence of a high-resolution turbulence model to a statistical steady state (fully developed turbulence). Second, in accurately and efficiently integrating turbulence models in which the detailed history of the small scales is not of interest. Finally, in accelerating the calculation of the statistics of a high-resolution turbulence simulation by calculating the statistics on the finest MTS level and using MTS to efficiently obtain different statistical regimes of the turbulent flow. Experience obtained in completely different computational problems indicates that the multigrid algorithm [3] is a most powerful tool from which turbulence studies may benefit as well. We hope the present work will serve as the first step in this direction.

$* * *$

We are grateful to Dr. R. MiLlifF for his help during the development of the twodimensional turbulence model, and to Drs. J. McWilliams, A. Brand'T and I. Procaccia for many useful discussions.

REFERENCES

[1] Vincent A. and Meneguzzi M., J. Fluid Mech., 225 (1991) 1; Zhen-Su She, Jackson E. and Orszag S. A., Nature, 344 (1990) 226; Santangelo P., Benzi R. and Legras B., Phys. Fluids A, 1 (1987) 1027; McWilliams J. C., J. Fluid Mech., 146 (1984) 21.

[2] Bryan F. O. and Holland W. R., in Parameterization of Small Scale Processes (Special Publication of the Institute of Geophysics, University of Hawaii, 1989) p. 99; SEMTNER J. A. and Chervin R. M. J., Geophys. Res., 93, C12, (1988) 15, 502, 522.

[3] BRANDT A., Multigrid Techniques: 1984 guide, Monograph, GMD-studie 85, GMD-FIT, Postfach 1240, D-5205, St. Augustin 1, Germany (1984).

[4] VoKe R. P., Technical report QMW EP-1082, Aeronautical Engineering, Queen Mary and Westfield College (1990).

[5] Sadourny R. and Basdevant C., J. Atmos. Sci., 42 (1985) 1353; Vallis K. G. and Hua B. L., J. Atmos. Sci., 45 (1988) 617. 